Revista Venezolana de Gerencia (RVG)

Año 10. № 30, 2005, 171-174

Universidad del Zulia (LUZ) • ISSN 1315-9984

\title{
EDITORIAL \\ Las Revistas Científicas de LUZ en la evaluación de méritos del FONACIT y en el PPI
}

Recientemente el Fondo Nacional de Ciencia, Tecnología e Innovación (FONACIT), realizó por tercera vez la evaluación de mérito de las revistas científicas incluidas en el Registro de Publicaciones Científicas y Tecnológicas Venezolanas en la convocatoria del año 2003 en esa institución, registro que también es producto de una evaluación, pero fundamentalmente centrada en el cumplimiento de la periodicidad.

La evaluación de mérito tiene el propósito de jerarquizar las revistas que se destaquen por la calidad de los artículos, la estructura de la revista, visibilidad e impacto, apertura a distintos contextos institucionales, calidad de los editores, y asesores, cumplimiento de normas e instrucciones de arbitraje y comportamiento editorial histórico. El resultado de esta evaluación constituye la base para el apoyo del FONACIT a las revistas, en el financiamiento de la edición, distribución, incorporación en el índice SCIELO, preparación de la versión electrónica y capacitación de los editores (Fuente: FONACIT).

Pero también el resultado constituye un insumo para que los editores avancemos en el mejoramiento de la calidad de los medios de difusión científica del país, por supuesto en la medida en que conozcamos detalles de este proceso de evaluación, por lo pronto sólo conocemos formalmente el título de las revistas que se ubicaron en el percentil cincuenta y setenta y cinco, ya que está publicado por la página web de la Fundación Venezolana de Promoción del Investigador, la cual administra el Programa de Promoción al Investigador (PPI) que acredita a los investigadores del país.

Participaron en la evaluación de méritos, un total de 93 de las 102 revistas científicas del país incorporadas al registro de publicaciones, es decir hubo 9 revistas que no se presentaron al proceso de evaluación de méritos. De estas 93, pertenecen a la Universidad del Zulia (LUZ), 22, el 23,6\%, ubicadas en distintas áreas del conocimiento, todas financiadas por el Consejo de Desarrollo Científico y Humanístico (CONDES) de LUZ. De estas 22 revistas, 13 pertenecen al campo de las ciencias sociales, de las cuales 10 obtuvieron más de 75 puntos sobre 100 en el proceso de evaluación, las otras tres quedaron por encima de los 50 puntos, una de ellas muy cerca de los 75 , con lo que queremos significar que las revistas de Ciencias Sociales de LUZ están muy bien calificadas en el FONACIT. La RVG fue la revista con la puntuación más alta en el campo de las ciencias sociales, no así de la totalidad de revistas, por encima de la RVG, con décimas de diferencia, estuvieron las siguientes revistas: Archivos Latinoamericanos de Nutrición, Bioagro, Zootecnia Tropical, Agronomía Tropical e INTERCIENCIA, revista multidisciplinaria de gran trayectoria, la cual obtuvo la puntuación más alta (Fuente: FONACIT). 
Editorial

Haydée Ochoa Henríquez

Del resto de las revistas de LUZ (nueve), tres obtuvieron más de 75 puntos y seis más de cincuenta, ninguna revista de LUZ estuvo por debajo de cincuenta puntos, significa que de las 22 revistas de esta institución, 13 estuvieron por encima de 75 puntos y el resto (nueve) por encima de 50, estas últimas en su mayoría cerca del percentil 75.

De las noventa y tres revistas evaluadas, 31 fueron ubicadas en el percentil 75, de las cuales 13 pertenecen a LUZ, es decir el 41,9\%. Considerando que participaron 30 instituciones, podríamos decir sin ánimo de competir, sino de buscar explicaciones al tema de los PPI el cual abordaremos en breve, que LUZ se ubica en primer lugar en la calidad de las publicaciones del país.

Es útil precisar que de las 93 publicaciones evaluadas, sólo seis están por debajo del percentil cincuenta, esto da cuenta de los esfuerzos que realizan las instituciones científicas y los editores del país, por disponer de revistas científicas de buena calidad. También es importante destacar que de las 93 revistas, 56 se ubican en el percentil 50, es decir el 60,2\% de las revistas están entre 50 y 75 puntos, es un dato que el FONACIT y las instituciones editoras deben considerar para la creación de estímulos en función de la necesidad de avanzar en la edición de revistas excelentes.

Por otra parte, actualmente se encuentra abierta la convocatoria de la Fundación Venezolana de Promoción de Investigador, que se realiza en junio todos los años, para acreditar a través de su PPI, a los investigadores que deseen ingresar y a aquéllos que les corresponde renovar para el siguiente año. El PPI es un programa con tres categorías: Candidato, Investigador y Emérito, dentro de la categoría de Investigador existen cuatro niveles, de inferior a superior, son los niveles I, II, III y IV. Los investigadores reciben un premio de acuerdo a la categoría y nivel donde son ubicados, la ubicación es realizada por pares organizados en Comisiones de Área por la Fundación. Al margen de la orientación de la investigación que ha promovido el PPI que por sus características, los investigadores, especialmente los de mucha trayectoria, miran más hacia fuera que hacia adentro, el programa se ha convertido una importante estrategia de estímulo a la investigación en el país.

En esta convocatoria, las publicaciones han sido clasificadas por el PPI como tipo A y B, las primeras son las revistas que se encuentran en algunos índices definidos por el programa y son clave para el ingreso y el ascenso al programa, Las revistas tipo B son aquellas que no estando en esos índices, además de ser arbitradas se encuentran en otros índices. Fueron calificadas al menos como tipo B las revistas clasificadas a partir del percentil cincuenta por el FONACIT. Todas las revistas de Ciencias Sociales de LUZ y casi todas las revistas financiadas por el CONDES son tipo A, ya que se encuentran registradas en los índices que constituyen requisito para calificarlas como tal.

Desde la convocatoria de 2003, LUZ viene ocupando el primer lugar entre los investigadores acreditados en el referido programa, de un total de 2684 investigadores acreditados, 656 pertenecían a LUZ, el mayor número por institución, esto representa un $24,44 \%$ del total de PPI (como se les llama a los investigadores acreditados), en un proceso donde participaron 22 instituciones, todas del Estado. Para este momento LUZ ocupó el segundo lugar (28,26\%), después de la Universidad Simón Bolívar que ocupó el primer lugar con un 32,84\% en la relación PPI/Profesores activos ordinarios a tiempo completo y dedicación exclusiva, que hicieron un total en LUZ de 2321 (Fuente: Fundación Venezolana de Promoción del Investigador).

En la convocatoria realizada en 2004, LUZ obtuvo 799 PPI, de un total de 3122, lo cual representa un $25,59 \%$, significa que incrementamos el porcentaje de investigadores acreditados en el programa y además la institución pasó al primer lugar en la relación PPI/personal, la cual fue de 36,91 por encima del $28,26 \%$ del año anterior, esta relación se incrementa por dos razones, aumentó el número de PPI y se redujo el número de profesores activos. 
Por qué se incrementa el número de PPI en LUZ?, son muchas las opiniones, quienes han pasado por la Dirección del CONDES lo atribuyen a las políticas de esta institución, algunos decanos que adversan al CONDES en su aspiración por la descentralización de los recursos de la investigación opinan que es un esfuerzo de los investigadores, independiente de las políticas institucionales, los editores opinamos que una explicación importante se encuentra en la calidad de las revistas, miembros de otras instituciones señalan que una gran mayoría de los PPI de LUZ son apenas candidatos, lo cual para otros es una fortaleza porque da cuenta de la formación de relevo. Estas y otras son sólo opiniones que se producen intentando buscar explicación a un fenómeno que ha llamado la atención de la comunidad científica del país.

No es propósito de este editorial explicar este fenómeno, pero sí asomar hipótesis que pudieran explorarse por quienes trabajan en tema de la gestión científica. Cuando se creó el PPI, LUZ decidió premiar a los investigadores que ingresaran a este programa, con una bonificación similar a la que otorga el PPI. En la mitad de los noventa, el vice-rectorado académico introduce algunos cambios para impulsar la investigación, dos cambios son clave, convertir el becario docente en becario académico con la obligación de hacer investigación y sacar a concurso la dirección del CON$D E S$, resultando ganador un investigador de trayectoria en publicaciones. A partir de este momento se sentaron las bases de un proceso de financiamiento a la investigación con base en la productividad científica publicada en revistas arbitradas e indizadas, esto tuvo lugar en un contexto en el cual se había creado el PPI, de esta forma LUZ se puso a tono con el PPI. Como parte de este proceso, todo financiamiento otorgado por el CONDES exige productividad en revistas arbitradas. A esto es necesario agregar que desde los noventa, se impulsan los doctorados en la institución, cuya demanda ha tomado fuerza en el último gobierno nacional, con las primas asignadas a los profesores que tengan concluido este nivel académico. La exigencia de productividad científica publicada en revistas arbitradas es una característica en la mayoría de los doctorados.

Se asume además desde este momento una política de financiamiento a revistas arbitradas, con variaciones en el tiempo. En los actuales momentos está garantizado el financiamiento de la edición, distribución y un asistente a las 22 revistas que han sido calificadas por el CONDES, sin posibilidad por problemas de recursos, de dar financiamiento a otras revistas de buena calidad que han surgido en los últimos años.

Es la única universidad del país que ha asumido como política, el financiamiento de las revistas de calidad, esto crea seguridad en los editores, para quienes ha quedado atrás la angustia que le produce tener que encontrar cada año una fuente de financiamiento de recursos no sólo adecuados en cantidad, sino también oportunos en el tiempo, lo que de alguna manera estimula a continuar avanzando en la producción editorial de excelente calidad. El financiamiento pasa por procesos de evaluación a través de informes anuales que deben presentar las revistas al solicitar financiamiento, con especial detenimiento en el cumplimiento de la periodicidad y evaluaciones periódicas al proceso y al producto. Algunas de estas revistas tienen además el apoyo de las facultades en infraestructura, es el caso de la Facultad de Ciencias Económicas y Sociales, donde se adscribe la RVG, cuestión que facilita el trabajo editorial.

Estamos así en presencia de políticas del gobierno, a través del PPI, de estímulo a la investigación y de políticas de LUZ cazadas con los criterios del PPI. Por otra parte se sientan las bases para que los nuevos ingresos de personal docente y de investigación, asuman ésta como parte de sus actividades.

A lo largo de este período, estas políticas se han ido afinando, con algunos altibajos, referencia especial merece el intento de la dirección del CONDES de no financiar las revistas en el 
Editorial

Haydée Ochoa Henríquez

año 2003, argumentando problemas de recursos, lo cual era cierto, pero al mismo tiempo haciendo sutilmente juego a los conflictos políticos del país, proceso en el cual los decanos aprovecharon para descentralizar parte importante de los recursos, debilitando de esta forma al CONDES. En estos altibajos está presente siempre la lucha de los editores, quienes cohesionados exigen a la institución dar continuidad a la política de financiamiento a las revistas.

A nuestro juicio cinco factores son clave en este proceso de incremento de los PPI en LUZ: Las políticas del CONDES, de financiamiento a la investigación, el premio de LUZ a los investigadores que ingresan al PPI, el financiamiento sistemático por parte del CONDES a las revistas, las cuales están a la vista de las nuevas generaciones con poca experiencia para moverse en el mundo de las publicaciones, la apertura de doctorados en la institución con exigencia de productividad científica y acompañada de primas por alcanzar este nivel y el trabajo editorial en manos de investigadores de trayectoria. Son factores que es necesario considerar seriamente a la hora de buscar explicación a este avance de LUZ en la investigación.

En el marco de estos éxitos de LUZ en el PPI y en la evaluación de mérito de las revistas por parte del FONACIT, editamos la RVG No. 30, correspondiente al segundo número del año diez. Iniciamos el trabajo editorial semestralmente, posteriormente la revista se editó cuatrimestralmente y hoy se edita trimestralmente. Ha sido un esfuerzo editorial de rigurosa periodicidad, financiado por el CONDES desde su origen.

En este número incorporamos siete trabajos, dos en LA MIRA, dedicada a LA GESTION URBANA. Por una parte, Sergio Paz de la Universidad Nacional de Quilmas (Argentina), presenta el trabajo titulado: Gestión Estratégica y Posicionamiento de Ciudades. La Marca de ciudad como política vector para la proyección internacional, Por otra parte, Rosa Virginia Ocaña de la Universidad Simón Bolívar, de Venezuela y Joheni Urdaneta de LUZ, presentan resultados de investigación sobre: Participación de los Municipios en la formación de la política nacional de transporte urbano en Venezuela. En la sección TRIMESTRE, tenemos cinco trabajos: Del investigador chileno Francisco Ganga de la Universidad de Los Lagos, el artículo titulado: Análisis preliminar del gobierno universitario chileno; de LUZ, de las investigadoras María Campo-Redondo y Marisela Árraga, el trabajo titulado: El Cine Foro como estrategia didáctica en la generación de ingresos universitarios; de la ULA, la investigadora Haydée Cecilia Rincón, publica el artículo titulado: Contabilidad de Costos y Gestión en la Industria Farmacéutica: Estudio de un caso; Oda Gómez de Angulo, Alira Chirinos González y Diego Herrera, de LUZ, nos entregan su trabajo titulado: Comportamiento de las firmas de Contadores Públicos en el Municipio Maracaibo y también de LUZ, Carmen Añez, publica el trabajo: El capital intelectual: Nuevo enfoque de la flexibilización Laboral. Publicamos además dos discursos del homenaje realizado a nuestro profesor y diputado a la Asamblea Nacional, el economista Rodrigo Cabezas: el discurso del homenajeado y el discurso del Dr. Jorge Chávez, decano de nuestra Facultad, son documentos que dan cuenta de la dinámica de nuestro país y nuestra universidad en tiempos de transformación.

Agradecemos a los autores su confianza en nuestra revista e invitamos a quienes están interesados en publicar en el futuro, a leer detenidamente las normas, el formato de arbitrajes y las instrucciones a los árbitros. Hemos decidido desde este año dar a conocer todos estos instrumentos a fin de que los autores potenciales conozcan a profundidad, cómo tiene lugar el proceso de evaluación de los trabajos, lo cual agiliza las relaciones con la RVG.

\section{Haydée Ochoa Henríquez*}

* Investigadora del Centro de Estudios de la Empresa de LUZ, editora de la Revista Venezolana de Gerencia. 